\title{
Active Vibration Control of the Sting Used in Wind Tunnel: Comparison of Three Control Algorithms
}

\author{
Xing Shen $\left(\mathbb{D},{ }^{1}\right.$ Yuke Dai $\left(\mathbb{D},{ }^{1}\right.$ Mingxuan Chen,, ${ }^{1,2}$ Lei Zhang, ${ }^{1}$ and Li Yu $\mathbb{D}^{3}$ \\ ${ }^{1}$ State Key Laboratory of Mechanics and Control of Mechanical Structures, Nanjing University of Aeronautics and Astronautics, \\ Nanjing 210016, China \\ ${ }^{2}$ Nanjing Chenguang Group Corporation Ltd., Nanjing 210006, China \\ ${ }^{3}$ China Aerodynamics Research and Development Center, Mianyang 621000, China \\ Correspondence should be addressed to Li Yu; 1492855418@qq.com
}

Received 23 May 2018; Accepted 1 August 2018; Published 20 September 2018

Academic Editor: Fabio Minghini

Copyright (C) 2018 Xing Shen et al. This is an open access article distributed under the Creative Commons Attribution License, which permits unrestricted use, distribution, and reproduction in any medium, provided the original work is properly cited.

\begin{abstract}
In wind tunnel tests, cantilever stings are often used as model-mount in order to reduce flow interference on experimental data. In this case, however, large-amplitude vibration and low-frequency vibration are easily produced on the system, which indicates the potential hazards of gaining inaccurate data and even damaging the structure. This paper details three algorithms, respectively, Classical PD Algorithm, Artificial Neural Network PID (NNPID), and Linear Quadratic Regulator (LQR) Optimal Control Algorithm, which can realize active vibration control of sting used in wind tunnel. The hardware platform of the first-order vibration damping system based on piezoelectric structure is set up and the real-time control software is designed to verify the feasibility and practicability of the algorithms. While the PD algorithm is the most common method in engineering, the results show that all the algorithms can achieve the purpose of over $80 \%$ reduction, and the last two algorithms perform even better. Besides, self-tuning is realized in NNPID, and with the help of the Observer/Kalman Filter Identification (OKID), LQR optimal control algorithm can make the control effort as small as possible. The paper proves the superiority of NNPID and LQR algorithms and can be an available reference for vibration control of wind tunnel system.
\end{abstract}

\section{Introduction}

In recent years, modern aircraft are gradually developing to light weight, high mobility, high speed, and high angle of attack, which apparently puts forward higher requirements for wind tunnel tests. And the quality of cantilever sting used in wind tunnel tests is the key point to obtain reliable data. However, when the model is exposed to aerodynamic loads from flow dynamic pressure, this flexible support system will easily produce low-frequency vibration and large-amplitude vibration, which will dramatically affect the accuracy of tests and even damage the structure. Therefore, in order to enhance the safety and accuracy of experiments, it is of vital importance to reduce the vibration of the sting.

National Transonic Facility (NTF) of NASA and the European Transonic Wind tunnel (ETW) were the first to start the research in related fields. The study by NTF began around 1990. In 1994, applying piezoelectric actuators as damping device, Wimmel [1] adopted a pure proportional control method to suppress the vibration of an F-18 aircraft model, and the experiment suppressed over $60 \%$ vibration amplitude. In 2007, Balakrishna et al. [1-3] proposed a scheme of embedding stacked piezoelectric actuators in the sting, and based on this thought, he had already developed three sets of vibration suppression systems until 2011. The related research in ETW can date back to 1996, and Hefer, in a patent, firstly proposed a device [4], which has six sets of piezoelectric ceramics encircled at the root or middle of the test sting to actively suppress the vibrations of the support system. On this basis, Fehren et al. [5] proposed an improved scheme in 2001 and increased the number of piezoelectric actuators to 14 .

Besides, some other organizations are also concerned about the vibration of wind tunnel models. Up to now, a great many of convincing results has been obtained in thousands of attempts [6-15]. And in term of algorithms, classical PD is still the most popular and practical control 
algorithm in engineering although it has some limitations, especially when the control objects are different, the parameters of the controller are difficult to adjust automatically to adapt to the changing of the external environment. Last decade witnesses the popularity of artificial intelligence, and the applications of neural networks have gradually replaced the traditional methods. The artificial neural network PID has set off a new trend, and many examples of success prove its superiority in parameter self-tuning. Besides, when the control effect reaches the standard, engineers began to focus on finding more economical and optimized control method. Therefore optimal control theory emerges as the times require, which has made great progress in both depth and breadth so far. LQR theory is one of the earliest and most mature methods for state-space design in modern control theory. LQR optimal control can make the original system achieve better performance index with cheap control effort.

Some other studies have illustrated different controllers for active vibration control purpose [16-21]; however, these two mentioned algorithms have not been widely used in wind tunnel tests yet. In order to introduce new applications of the advanced algorithms, this paper presents the details of the design of PD, NNPID, and LQR controllers. Besides, the hardware platform of the vibration damping system is set up, and the real-time control software is designed to verify the feasibility and practicability of the whole system. The ground experiments, under the excitation of the exciter are carried out, and the effects of the three algorithms are compared. The results show the availability and superiority of the methods, which lays a foundation for the application in follow-up wind tunnel tests.

\section{Concepts and Approaches}

2.1. Structure of the System. In wind tunnel tests, the support system which is composed of a cantilever sting, a test balance and a model is exposed to wide frequency aerodynamic load, including static load and dynamic load, and the load produces dynamic bending along any section of the sting. In fact, the bending moment in pitch direction is much larger than that of the other two directions, so the primary target is to suppress the vibration in the direction of pitching.

The system can be regarded as a mass spring system with multiple degrees of freedom and low damping. Under aerodynamic loads, the vibration equation of the system can be expressed as

$$
M \ddot{x}+C \dot{x}+K x=F,
$$

where $M$ is the mass matrix of the system, $C$ is the damping matrix, $K$ is the stiffness matrix, $F$ is the force vector of the system, and $x$ represents the displacement vector.

A piezoelectric actuator is applied to realize vibration control, the principle of the structure is shown in Figure 1: the piezoelectric stack is installed at the bottom of the sting and is actuated by control voltage, and then restoring moment is generated to offset the effect of loading moment. Thus, the vibration suppression in the direction of pitching is realized.

Figure 2 shows the structure of vibration damping system. When the sting begins to vibrate by aerodynamic load, the test balance together with the strain gauge begins to transform the mechanical signal into voltage signal. After smoothing by a low pass filter, the signal is sent to the controller. Then, the control signal is amplified by a power amplifier to generate the driving voltage. Finally, the piezoelectric stack is actuated by the driving voltage to suppress the vibration of the sting. Thus, a negative feedback control system is formed.

2.2. Classical PD Algorithm. In order to realize PD control, the rational fraction polynomial fitting (levy) method is used to fit the transfer function according to the frequency response function of the vibration system, and the modal parameters such as frequency, damping ratio, and mode shape are obtained. Then, the PD control algorithm is proposed based on the fitted system function.

2.2.1. Levy Method. Rational fraction polynomial method, also known as levy method, is one of the most widely used frequency domain identification methods in engineering. The basic idea is to fit the theoretical transfer function to the measured frequency response curve and minimize the error. In the process of recognition, a target function $J$ is introduced to linearize the error function:

$$
\begin{array}{r}
J=\sum_{i=1}^{N}\|A(j \omega) \mathcal{\varepsilon}(j \omega)\|^{2}=\sum_{i=0}^{N}\left\|H\left(j \omega_{i}\right) A\left(j \omega_{i}\right)-B\left(j \omega_{i}\right)\right\|^{2} \\
i=1,2, \ldots, N .
\end{array}
$$

As for the experiment, first-order mode of the sting contributes most to vibration. According to the theory of single mode vibration, the model is a second-order system: $G(s)=(B(s)) /(A(s))=\left(b_{0}+b_{1} s\right) /\left(1+a_{1} s+a_{2} s^{2}\right)$. In order to find the coefficients $a_{1}, a_{2}, b_{0}, b_{1}$, set the partial derivative of the equation (2) to zero, and the following equation can be found:

$$
\left[\begin{array}{cccc}
V_{0} & 0 & T_{1} & S_{2} \\
0 & V_{2} & -S_{2} & T_{3} \\
T_{1} & -S_{2} & U_{2} & 0 \\
S_{2} & T_{3} & 0 & U_{4}
\end{array}\right]\left[\begin{array}{l}
b_{0} \\
b_{1} \\
a_{1} \\
a_{2}
\end{array}\right]=\left[\begin{array}{c}
S_{0} \\
T_{1} \\
0 \\
U_{2}
\end{array}\right],
$$

where

$$
\begin{aligned}
V_{j} & =\sum_{i=0}^{N} \omega_{i}^{j}, \\
S_{j} & =\sum_{i=0}^{N} \omega_{i}^{j} R\left(\omega_{i}\right), \\
T_{j} & =\sum_{i=0}^{N} \omega_{i}^{j} I\left(\omega_{i}\right), \\
U_{j} & =\sum_{i=0}^{N} \omega_{i}^{j}\left[R\left(\omega_{i}\right)+I\left(\omega_{i}\right)\right] .
\end{aligned}
$$

The system transfer function can be obtained by calculating the parameters in the matrix. And final result for the damping system is 


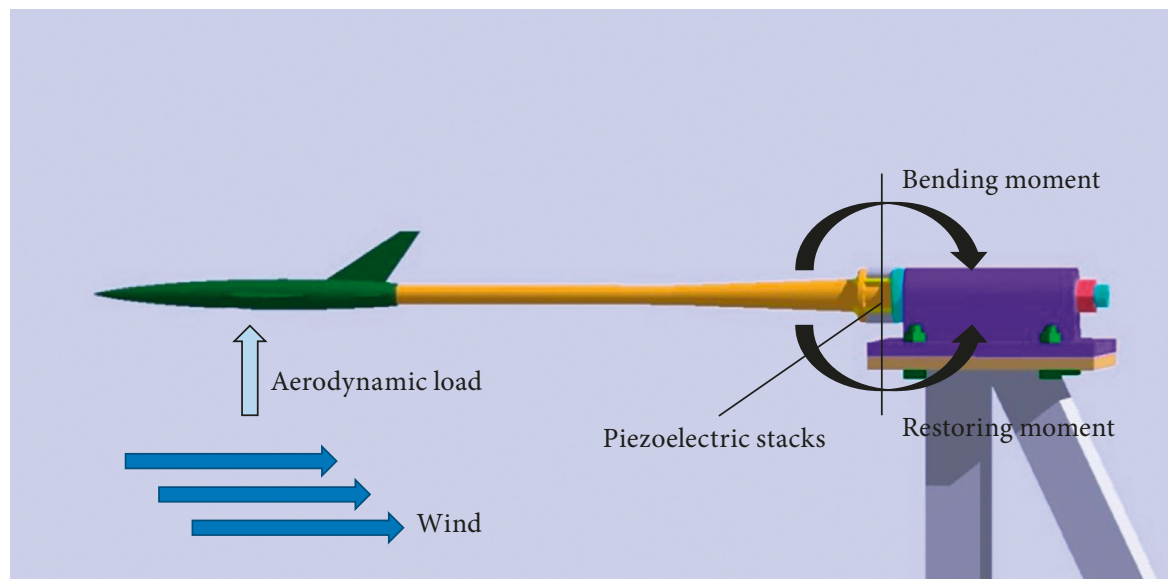

Figure 1: Diagram of the cantilever sting system.

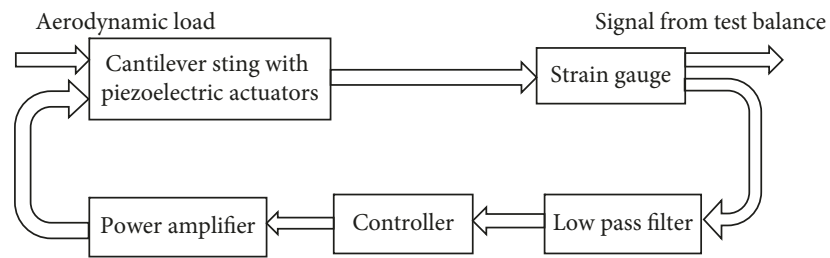

FIGURE 2: Diagram of vibration damping system.

$$
G(s)=\frac{-0.0016876+3.8586 \times 10^{-5} s}{1+1.1355 \times 10^{-4} s+2.1276 \times 10^{-4} s^{2}} .
$$

2.2.2. Controller Design. PID (proportion, integral, and derivative) controller, as the earliest practical controller, has been used for nearly a hundred years and is still the most widely used industrial controller. Figure 3 shows the main diagram of digital PID controller.

The mathematical expression of the PID controller is

$$
u(t)=K_{\mathrm{P}}\left[e(t)+\frac{1}{T_{\mathrm{I}}} \int_{0}^{t} e(\tau) \mathrm{d} \tau+T_{\mathrm{D}} \frac{d e(t)}{d t}\right] .
$$

Its transfer function is $G_{\mathrm{PID}}(s)=K_{\mathrm{P}}\left(1+\left(1 /\left(T_{\mathrm{I}} s\right)\right)+\right.$ $\left.T_{\mathrm{D}} s\right)=K_{\mathrm{P}}+\left(K_{\mathrm{I}} / s\right)+K_{\mathrm{D}} s$. Because computer control is a discrete control, it can only work according to the deviation value of each sampling time. By backward finite differences, the discrete transfer function can be obtained:

$$
\begin{aligned}
u(k) & =K_{\mathrm{P}}\left[e(k)+\frac{T}{T_{\mathrm{I}}} \sum_{j=0}^{k} e(j)+T_{\mathrm{D}} \frac{e(k)-e(k-1)}{T}\right] \\
& =K_{\mathrm{P}} e(k)+K_{\mathrm{I}} \sum_{j=0}^{k} e(j)+K_{\mathrm{D}}[e(k)-e(k-1)] .
\end{aligned}
$$

Due to the characteristics of the vibration system, the structure will turn back to the original equilibrium point after attenuation, so there is no need to consider the steady-state error. Therefore, the proportional derivative

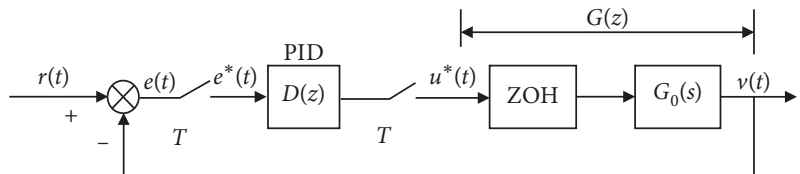

FIgURE 3: Diagram of digital PID.

(PD) control is adopted and the transfer function can be simplified as

$$
G_{\mathrm{C}}(s)=K_{\mathrm{P}}+K_{\mathrm{D}} s .
$$

Since the characteristic signal controlled by the system is a sinusoidal signal, it is necessary to calibrate the phase, especially the phase at the natural frequency. In engineering, a common method of phase correction is to set $\angle G_{\mathrm{C}}\left(j \omega_{r}\right)=$ $-\angle G\left(j \omega_{r}\right)$, where $\omega_{r}$ is the first-order frequency. Therefore the ratio and differential parameters of the PD controller should meet the following relation:

$$
\begin{aligned}
\sqrt{K_{\mathrm{P}}^{2}+\left(\omega_{r} K_{\mathrm{D}}\right)^{2}} & =\frac{(1 / g)-1}{\left|G\left(j \omega_{r}\right)\right|}, \\
\arctan \frac{\omega_{r} K_{\mathrm{D}}}{K_{\mathrm{P}}} & =-\angle G\left(j \omega_{r}\right)+\varphi .
\end{aligned}
$$

where $g$ represents the desired amplitude ratio of input and output voltages while $-\angle G\left(j \omega_{r}\right)+\varphi$ represents the desired phase difference. By manually adjusting the two parameters, different results of PD parameters can be gained.

2.3. NNPID Algorithm. Among a large number of neural networks, back-propagated network (BP-NN), whose structure is clear and the learning method is simple, has the ability to fit any nonlinear function and is gradually developing to mature nowadays. Therefore, this paper adopts self-learning PID control algorithm based on BP neural network, and its structure is shown in Figure 4.

This paper uses adaptive linear neuron model, and the supervised error correction learning is realized by the gradient descent (Delta rule). The formula for the classical PID is $u(k)=K_{\mathrm{P}}(k) e_{\mathrm{P}}(k)+K_{\mathrm{I}}(k) e_{\mathrm{I}}(k)+K_{\mathrm{D}}(k) e_{\mathrm{D}}(k)$. Where 


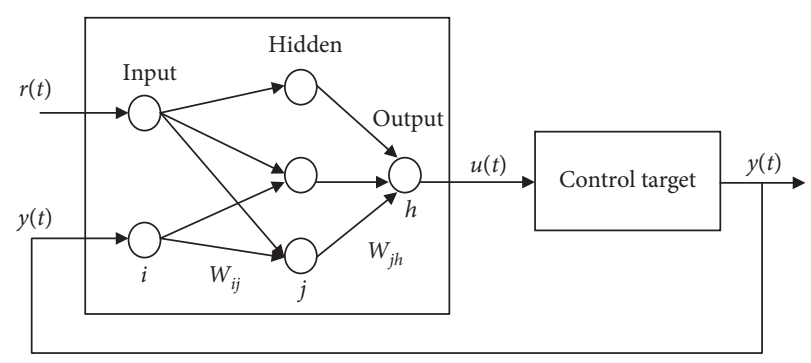

FIGURE 4: Schematic diagram of a BP-NN controller.

$$
\left\{\begin{array}{l}
e_{\mathrm{P}}(k)=r(k)-y(k)=-y(k), \\
e_{\mathrm{I}}(k)=\sum_{j=1}^{k} e_{\mathrm{P}}(j)=-\sum_{j=1}^{k} y(j), \\
e_{\mathrm{D}}(k)=e_{\mathrm{P}}(k)-e_{\mathrm{P}}(k-1)=-[y(k)-y(k-1)] .
\end{array}\right.
$$

Self-tuning is realized according to delta learning rules so that the recursive formula of weight value is

$$
\left\{\begin{array}{l}
K_{\mathrm{P}}(k+1)=K_{\mathrm{P}}(k)+\beta \frac{\partial \varepsilon}{\partial K_{\mathrm{P}}}, \\
K_{\mathrm{I}}(k+1)=K_{\mathrm{I}}(k)+\beta \frac{\partial \varepsilon}{\partial K_{\mathrm{I}}}, \\
K_{\mathrm{D}}(k+1)=K_{\mathrm{D}}(k)+\beta \frac{\partial \varepsilon}{\partial K_{\mathrm{D}}} .
\end{array}\right.
$$

All the three weights use the same learning rate, and according to the chain rule, Equation (12) is established:

$$
\left\{\begin{array}{c}
\frac{\partial \varepsilon}{\partial K_{\mathrm{P}}}=\frac{\partial \varepsilon}{\partial y(k+1)} \cdot \frac{\partial y(k+1)}{\partial u(k)} \cdot \frac{\partial u(k)}{\partial K_{\mathrm{P}}} \\
\frac{\partial \varepsilon}{\partial K_{\mathrm{I}}}=\frac{\partial \varepsilon}{\partial y(k+1)} \cdot \frac{\partial y(k+1)}{\partial u(k)} \cdot \frac{\partial u(k)}{\partial K_{\mathrm{I}}} \\
\frac{\partial \varepsilon}{\partial K_{\mathrm{D}}}=\frac{\partial \varepsilon}{\partial y(k+1)} \cdot \frac{\partial y(k+1)}{\partial u(k)} \cdot \frac{\partial u(k)}{\partial K_{\mathrm{D}}}
\end{array}\right.
$$

where

$\left\{\begin{array}{l}\partial \varepsilon /(\partial y(k+1))=(\partial[-y(k+1)]) /(\partial y(k+1))=-1 \\ (\partial y(k+1)) /(\partial u(k))=(y(k+1)-y(k)) /(u(k)-u(k-1))\end{array}\right.$ and because the change of $[u(k)-u(k-1)]$ is small, the equation $(\partial y(k+1)) /(\partial u(k))=\operatorname{sgn}[(y(k+1)-y(k)) /$ $(u(k)-u(k-1))]$ is workable. Finally, the self-learning formula of neural network PID is obtained:

$$
\left\{\begin{array}{l}
K_{\mathrm{P}}(k+1)=K_{\mathrm{P}}(k)-\beta \operatorname{sgn}\left[\frac{y(k+1)-y(k)}{u(k)-u(k-1)}\right], \\
K_{\mathrm{I}}(k+1)=K_{\mathrm{I}}(k)-\beta \operatorname{sgn}\left[\frac{y(k+1)-y(k)}{u(k)-u(k-1)}\right], \\
K_{\mathrm{D}}(k+1)=K_{\mathrm{D}}(k)-\beta \operatorname{sgn}\left[\frac{y(k+1)-y(k)}{u(k)-u(k-1)}\right] .
\end{array}\right.
$$

2.4. LQR Algorithm. The optimal control method, as shown in Figure 5, is one of the most complete control algorithms in the modern control method theory system. In this section, the Linear Quadratic Regular (LQR) control algorithm is used to design the controller for active vibration control.

2.4.1. The Observer/Kalman Filter Identification Method. In order to design the effective LQR controller, the state-space model of the system is essential. This paper adopts the Observer/Kalman Filter Identification (OKID) method together with Eigensystem Realization Algorithm (ERA) to get the state-space equation. OKID, which is proposed by Juang et al. [22, 23] and used convergence rate of the Markov parameters of the asymptotically stable observer acceleration system, has advantages of a little computational effort and high accuracy. Its process is as follow: firstly, obtain input and output data of the system by sampling. Secondly, calculate the Markov parameters $\bar{Y}$ of the observer. Thirdly, according to equation (14), use $\bar{Y}$ to calculate Markov parameter sequence $Y$ of the computing system.

$$
\begin{aligned}
D & =Y_{0}=\bar{Y}_{0} \\
Y_{k} & =\bar{Y}_{k}^{(1)}-\sum_{i=1}^{k} \bar{Y}_{i}^{(2)} Y_{k-i}, \quad k=1, \ldots, p, \\
Y_{k} & =-\sum_{i=1}^{p} \bar{Y}_{i}^{(2)} Y_{k-i}, \quad k=p+1, \ldots, \infty
\end{aligned}
$$

Finally, the ERA is implemented. The algorithm begins by forming the block $l \times l$ Hankel matrix $H$ which is given by

$$
H(l, i)=\left[\begin{array}{cccc}
Y_{i} & Y_{i+1} & \cdots & Y_{i+l-1} \\
Y_{i+1} & Y_{i+2} & \cdots & Y_{i+l} \\
\vdots & \vdots & \ddots & \vdots \\
Y_{i+l-1} & Y_{i+l} & \cdots & Y_{i+2 l-2}
\end{array}\right] .
$$

Defining a $q \times l q$ matrix $E_{q}^{T}=\left[I_{q}, 0\right]$ and an $m \times l m$ matrix $E_{m}^{T}=\left[I_{m}, 0\right]$ made up of identity and null matrices of the form. And then a discrete-time minimal order realization of the system can be written as

$$
\begin{aligned}
& A=\sum_{n}^{-1 / 2} U_{n} H(l, 1) V_{n} \sum_{n}^{-1 / 2}, \\
& B=\sum_{n}^{-1 / 2} V_{n}^{T} E_{m}, \\
& C=E_{q}^{T} U \sum_{n}^{-1 / 2},
\end{aligned}
$$




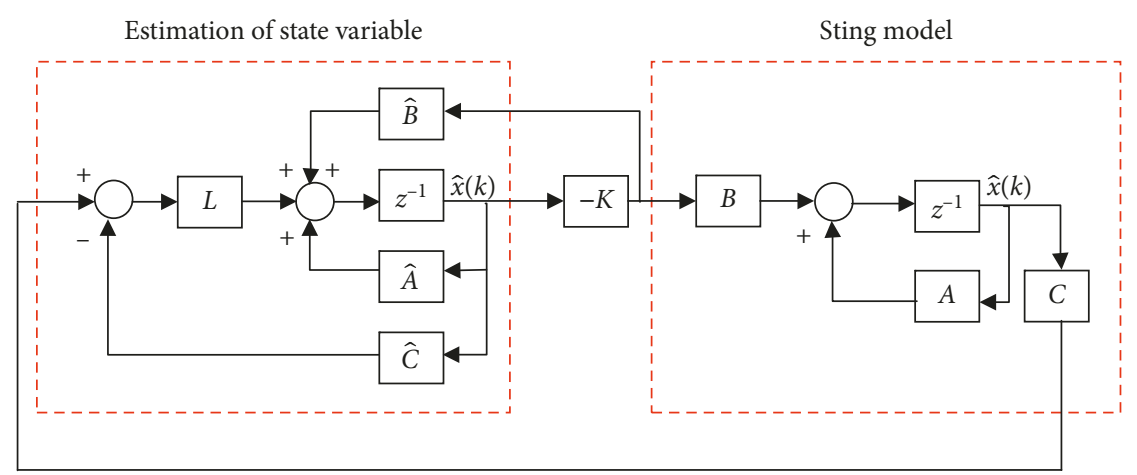

FIGURE 5: Diagram of a LQR controller.

where the matrices $U$ and $V$ are unitary matrices, $\sum$ is an $n \times n$ diagonal matrix of positive singular values, and $n$ is the order of the system. And after calculation, the identified system is given as follows

$$
\begin{aligned}
A & =\left[\begin{array}{ccc}
0.01642 & 0.06444 & 0.13977 \\
-0.06444 & 1.00243 & -0.03425 \\
0.13977 & -0.03425 & 0.97907
\end{array}\right], \\
B & =\left[\begin{array}{c}
0.11055 \\
-0.00482 \\
-0.00303
\end{array}\right], \\
C & =\left[\begin{array}{lll}
-0.11055 & -0.00482 & 0.00303
\end{array}\right], \\
D & =-0.01221 .
\end{aligned}
$$

2.4.2. Controller Design. Define the quadratic performance index of the system:

$$
J=\sum_{i=1}^{\infty}\left[x^{T}(i) Q x(i)+u^{T}(i) R u(i)\right],
$$

where the weighted matrix $Q$ is a symmetric semidefinite matrix and $R$ is a symmetric positive definite matrix. It is worth mentioning that $x^{T}(i) \mathrm{Q} x(i)$ expresses the comprehensive error between the given state and the actual state of the system in the whole process of control, namely, the extent to which the state variable $x(k)$ of the system leaves its equilibrium position, thus it reflects the control effect. And $U^{T} R(k) U$ is related to the control vector $U(k)$, which describes the energy output in the whole control process and reflects the cost of control. It is obvious from equation (18) that these two integral terms are restricted to each other. Therefore, the minimum value of the sum of two integral terms is essentially the compromise of the control effect and the control effort in optimal sense.

The solution of the optimal control is

$$
u(k)=-K x(k),
$$

where $K$ is a state feedback matrix, satisfying $K=(R+$ $\left.B^{T} P B\right)^{-1} B^{T} P A$, where $P$ is a positive definite matrix and satisfies the Riccati equation: $A^{T} P A-P-A^{T} P B(R+$ $\left.B^{T} P B\right)^{-1} B^{T} P A+Q=0$.
2.5. Experimental Setup. In order to verify the effectiveness of the control algorithm, a cantilever sting used in wind tunnel is processed and a measurement and control system is built. As shown in Figures 6(a) and 6(b), the front section of the sting is fitted with a test balance to obtain voltage signal aroused by exciter. The first-order natural frequency of the system is about $13.6 \mathrm{~Hz}$, so that the exciter releases mechanical vibration of the same frequency to simulate the resonance situation in wind tunnel.

When the sting begins to vibrate, the strain signal in the balance emerges and starts to transmit by the wires. After handling by a strain gauge and an antialiasing filter, the signal is sent to the controller, where the control signal is calculated according to the algorithm formula. Then, the power amplifier works to amplify the control signal. At last, the final control signal is transferred to the piezoelectric stack at the bottom of the sting to suppress vibration.

Figure 6(c) outlines the construction of the piezoelectric device. This is a bilaterally symmetric structure with a pair of piezoelectric stacks fixed at the root. Thanks to reverse piezoelectric effect, the tips of the stacks will stretch slightly when external voltage is exerted and simultaneously push the surface of a flexure hinge, which is used to amplify the displacement so that the restoring moment is produced on the cantilever sting.

The control algorithm is programmed in LabVIEW2012. And a National Instruments-produced PXI-7841R board card is used to collect and release signal, which possesses 8 analog input and output channels and has a FPGA module. Thus, the hardware platform can achieve high speed acquisition so that it ensures the least delay of time.

\section{Results and Comparison}

3.1. Results of PD Algorithm. In the experiment, a vibration exciter is used to generate the vibration near the resonance frequency of the sting. By manually changing the parameters of the controller, namely $g$ and $-\angle G\left(j \omega_{r}\right)+\varphi\left(\angle G\left(j \omega_{1}\right)=\right.$ $\left.-9.2^{\circ}\right)$ in equation (9), different results can be gained. When fixing $\varphi$ at 0 and changing the value of $g$, A group of the experimental results is shown in Table 1 . In that case, $K_{\mathrm{P}}$ and $K_{\mathrm{D}}$ are dramatically changing with the varying of $g$, and the effect of control is negatively related to the value of $g$. 


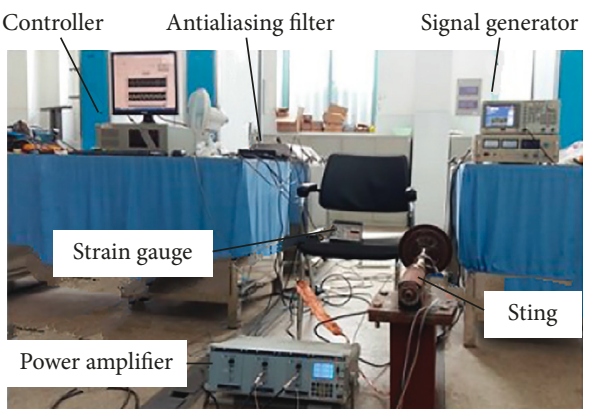

(a)

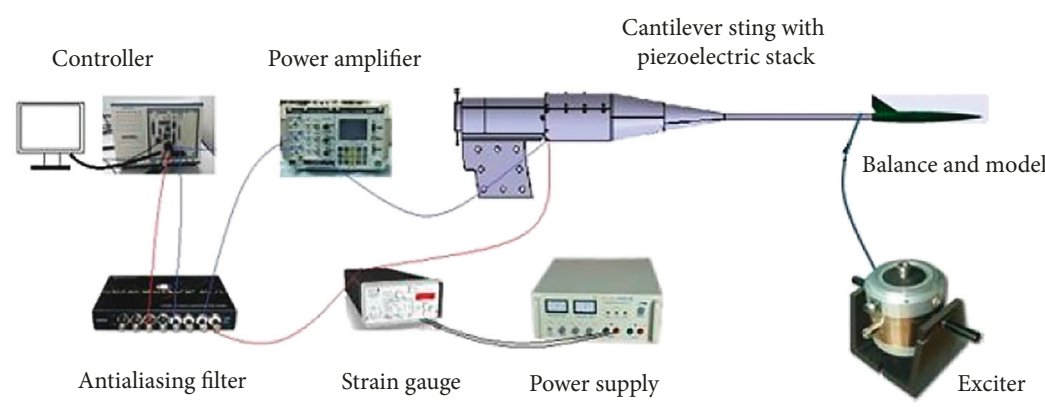

(b)

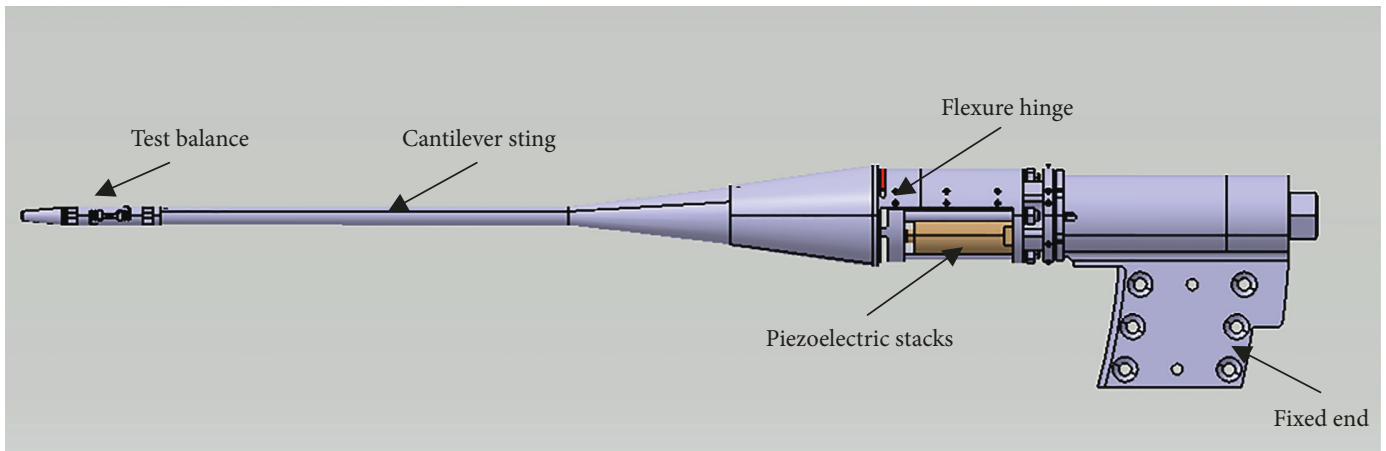

(c)

Figure 6: (a) The composition of the active vibration control system: (b) wiring diagram of the active vibration control system and (c) the figure of the cantilever sting with piezoelectric stacks.

TABLE 1: Comparison of vibration damping results with changing $g$.

\begin{tabular}{lcccc}
\hline$g$ & $K_{\mathrm{P}}$ & $K_{\mathrm{D}}$ & $\begin{array}{c}\text { Average p-p } \\
\text { value }(\mathrm{mV})\end{array}$ & $\begin{array}{c}\text { Percentage } \\
\text { of damping }\end{array}$ \\
\hline 1 & 0 & 0 & 503.28 & 0 \\
0.5 & -0.964 & -3.62766 & 257.17 & $48.9 \%$ \\
0.4 & -1.446 & -5.44149 & 210.23 & $58.23 \%$ \\
0.3 & -2.2493 & -8.46455 & 167.8 & $66.7 \%$ \\
0.2 & -3.856 & -14.5107 & 96.32 & $79.33 \%$ \\
0.1 & -8.676 & -32.649 & Fail & - \\
\hline
\end{tabular}

However, when $g$ is too small, the algorithm would defect, so it is worth paying attention to choose suitable value of $g$.

Table 2 shows a group of experimental results when $g$ is fixed at 0.2 while the value of $-\angle G\left(j \omega_{r}\right)+\varphi$ is changing. It is easy to find that the peak-peak values change with the changing of $\varphi$; however, the figures change slightly, and the results diverge when the value deviates far, which indicates that the influence of parameter $g$ is larger than that of $\varphi$.

After all, the results prove the stability and reliability of the algorithm. And the best result was obtained when $g$ is equal to 0.1 and $-\angle G\left(j \omega_{\mathrm{r}}\right)+\varphi$ is $-20^{\circ}$, and in that case, the peak-to-peak value of the signal declines from $503.28 \mathrm{mV}$ to $94.02 \mathrm{mV}$, and the power spectrum declines from $-15.01 \mathrm{~dB} / \mathrm{Hz}$ to $-38.12 \mathrm{~dB} / \mathrm{Hz}$ which indicates that more than $81 \%$ reduction of displacement response has been achieved.

3.2. Results of NNPID Algorithm. The most appealing advantage of NNPID algorithm is the ability of self-tuning. As
TABLE 2: Comparison of vibration damping results with changing $\varphi$.

\begin{tabular}{lccc}
\hline$-\angle G\left(j \omega_{\mathrm{r}}\right)+\varphi$ & $K_{\mathrm{P}}$ & $K_{\mathrm{D}}$ & Average $\mathrm{p}-\mathrm{p}$ value $(\mathrm{mV})$ \\
\hline-30 & -3.3829 & -45.3795 & 108.21 \\
-20 & -3.67067 & -31.0414 & 94.02 \\
-10 & -3.84691 & -15.7601 & 98.23 \\
-5 & -3.90002 & -5.12393 & 100.03 \\
0 & -3.90625 & 0 & 101.65 \\
+5 & -3.90002 & +5.12393 & 106.41 \\
+10 & -3.84691 & +15.7601 & Fail \\
+20 & -3.67067 & +31.0414 & Fail \\
+30 & -3.3829 & +45.3795 & Fail \\
\hline
\end{tabular}

shown in Figure 7, when appropriate learning rate is set, the controller is supposed to find the most suitable parameters automatically. It is worth noting that the learning rate plays an important role. Table 3 provides the performance of NNPID controller with different value of $\beta$ : the overlarge learning rate might cause the control parameters to constantly change, which makes the control signal unstable and finally leads to failure. Otherwise, the time is longer and the effect of control will reduce.

In the experiment, the best result is gained when the learning rate $\beta$ is set at 0.016 , and the time-domain signal and power spectrum of which are demonstrated in Figures 8 and 9 , respectively. It is easy to find that with the NNPID control on, the peak-to-peak value of the signal declines from $502.12 \mathrm{mV}$ to $58.24 \mathrm{mV}$, and the power spectrum declines from $-14.91 \mathrm{~dB} / \mathrm{Hz}$ to $-33.32 \mathrm{~dB} / \mathrm{Hz}$. More than $88 \%$ reduction of vibration has been achieved which 

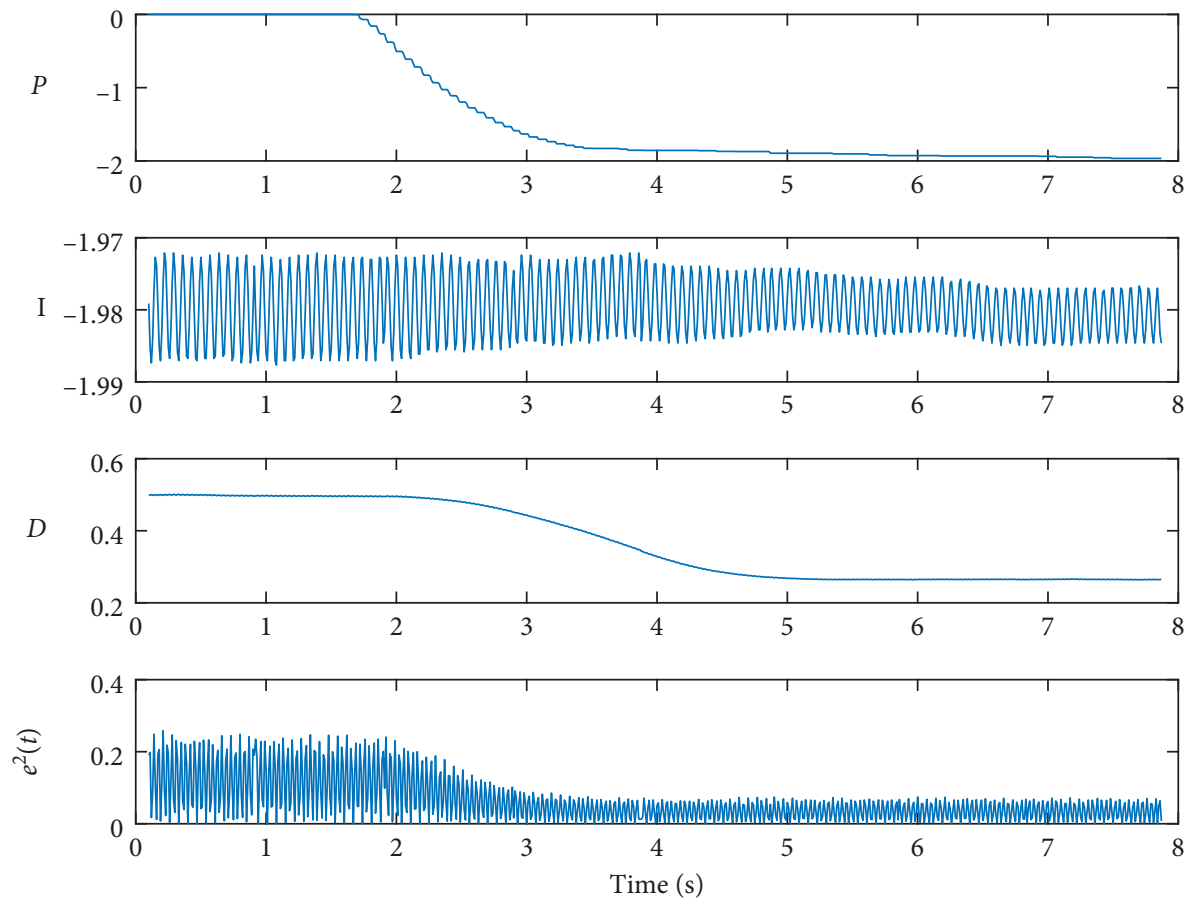

Figure 7: Self-tuning process of PID parameters.

TABLE 3: Comparison of vibration damping results with changing $\beta$.

\begin{tabular}{lcc}
\hline$\beta$ & Time of self-tuning $(\mathrm{s})$ & $\mathrm{p}-\mathrm{p}$ value $(\mathrm{mV})$ \\
\hline 0.001 & 15.4 & 90.12 \\
0.01 & 3.4 & 65.78 \\
0.016 & 1.2 & 58.24 \\
0.02 & 0.5 & 70.09 \\
0.05 & 0.1 & 208.19 \\
0.1 & Fail & Fail \\
\hline
\end{tabular}

indicates that the sting is effectively controlled under continuous load.

3.3. Results of LQR Algorithm. As far as LQR controller is considered, in order to get the optimal result in the experiment, the selection of parameter $Q$ and $R$ is of vital importance. Since $Q$ determines the importance of the control performance and $R$ determines that of the control effort, when the control performance is satisfactory, it is necessary to set $R$ relatively small to save energy. The optimal values for $Q$ and $R$ are typically obtained by trial-and-error. Generally, $R$ is determined by $I$ and $\rho$, where $\rho$ is a scalar parameter. Thus, the choosing of $Q$ and $R$ is realized by changing the value of $\rho$. In this experiment, the best result comes out when $\rho$ is below 1 . And several experimental data are shown in Table 4.

When $\rho$ is equal to 0.04 and the control gain matrix $K$ was calculated using to give $K=\left[\begin{array}{lll}0.69971 & -5.21489 & 2.69427\end{array}\right]$, the $\mathrm{p}-\mathrm{p}$ value of the output fell to a minimum, which represents the best result in this experiment.

Figures 10 and 11 show the diagrams of the best result. It is easy to find that with the LQR control on, the peak-to-peak value of the signal declines from $503.86 \mathrm{mV}$ to $69.4 \mathrm{mV}$ and the power spectrum declines from $-13.11 \mathrm{~dB} / \mathrm{Hz}$ to $-29.33 \mathrm{~dB} / \mathrm{Hz}$. More than $86 \%$ reduction of vibration is realized. Furthermore, due to the characteristics of optimal control theory, when the control effect is guaranteed, the output power during the whole process ought to be the least, that is to say, the experiment realizes the least of control effort.

3.4. Comparison of Three Control Algorithms. As shown in Figure 5, the results indicate that all the three algorithms can effectively achieve the goal of reducing vibration. As the most widely used method in engineering, PD algorithm achieves $81 \%$ of vibration damping in $1.5 \mathrm{~s}$. The principle of $\mathrm{PD}$ is simple, and its application is already mature. However, once the structure changes, the repetition for system identification processing is in dispensable in order to find available control parameters, which is inconvenient and time-wasting.

The experiments prove the feasibility and advancement of NNPID algorithm. By finding the most appropriate learning rate, NNPID can realize the self-tuning of the control parameters. Different from the traditional PD algorithm, there is no need to launch system identification processing in NNPID, which means a significant convenience for different engineering occasions. Besides, the NNPID algorithm achieves the largest percentage of vibration damping (88\%) in $1.2 \mathrm{~s}$, which is also faster than PD algorithm (Table 5).

And on the premise of guaranteeing the effect of control, LQR optimal control algorithm shows the highest costsaving ability, though the one of the most obvious drawbacks 


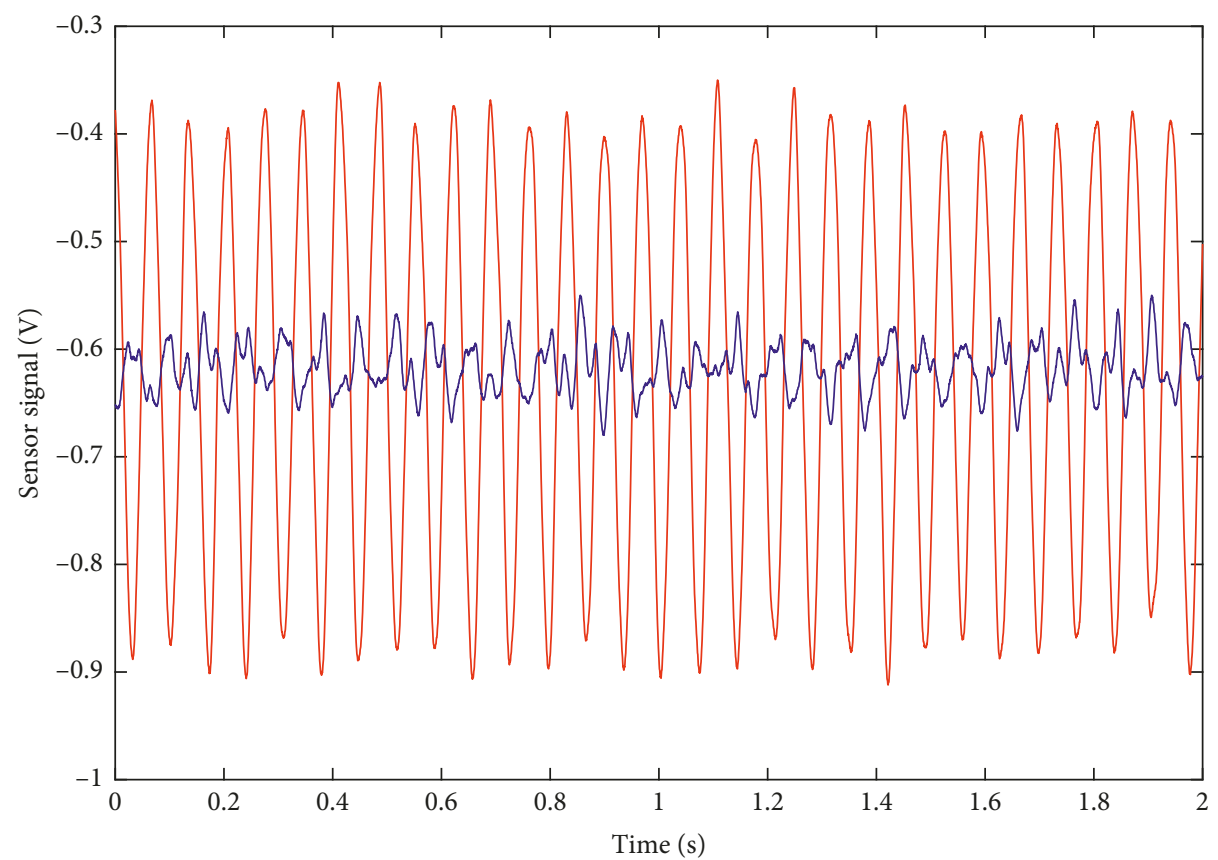

- Control OFF

- Control ON

FIgURE 8: Comparison of time-domain signal with NNPID control on/off.

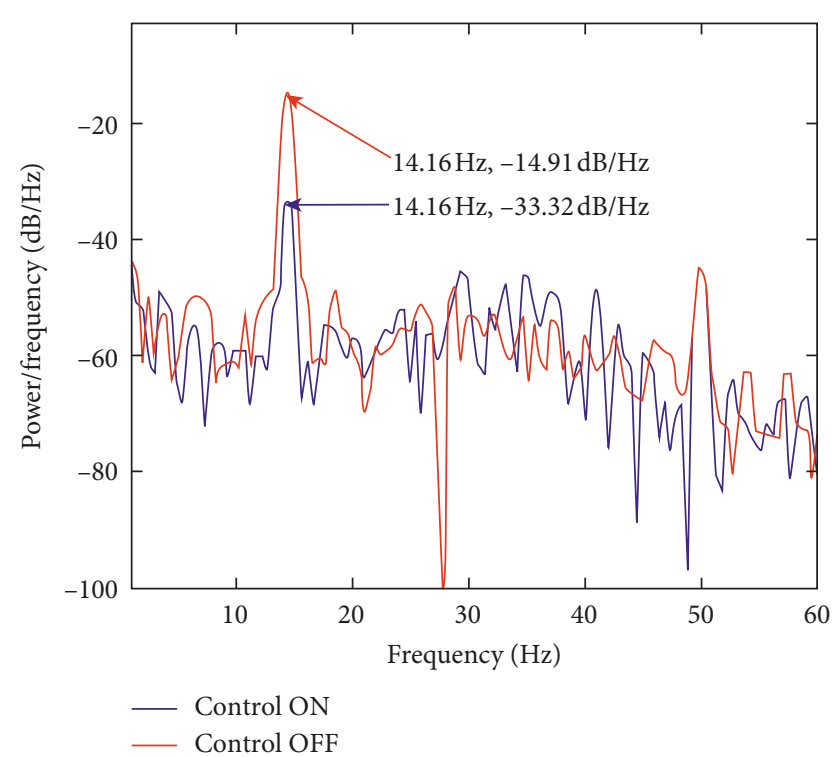

FIGURE 9: Comparison of power spectrum with NNPID control on/off.

of LQR is that the modeling process is complicated and many of the parameters need to be set. However, it has the potential to work in large power consumption conditions or power-limited situations, like in high-power wind tunnels, which saves a huge amount of energy.

\section{Conclusions}

In this paper, a wind tunnel cantilever sting, with a pair of piezoelectric actuators embedded at the end is processed,
TABLE 4: Comparison of vibration damping results with changing $\rho$.

\begin{tabular}{lc}
\hline$\rho$ & $\mathrm{p}-\mathrm{p}$ value $(\mathrm{mV})$ \\
\hline 0.02 & 66.49 \\
0.04 & 65.29 \\
0.06 & 65.31 \\
0.08 & 66.90 \\
0.1 & 67.89 \\
\hline
\end{tabular}

and a measurement and control system based on LabVIEWFPGA module is built to verify the effectiveness of three algorithms. A classical PD, a NNPID, and a LQR controller have been designed in detail. In active damping evaluation tests, it is shown that, for all the controllers, over than $80 \%$ of displacement response of the sting mode can be eliminated. Typically, the NNPID and LQR even achieve nearly $90 \%$ reduction of vibration. The success of the experiments proves new application area of the algorithms, and it foreshadows innovative methods for wind tunnel test, which can be an available reference for wind tunnel study.

Need to say, the experiments in this paper contribute to reduce the vibration of only the first order of the system. Results could be better when the order increases, which would be the deeper research in the future.

\section{Data Availability}

The experimental data used to support the findings of this study may be released upon application to the Yuke Dai, who can be contacted at ykdai@nuaa.edu.cn. The details of the structure used to support the findings of this study (including the balance, the model, and the sting) were supplied 


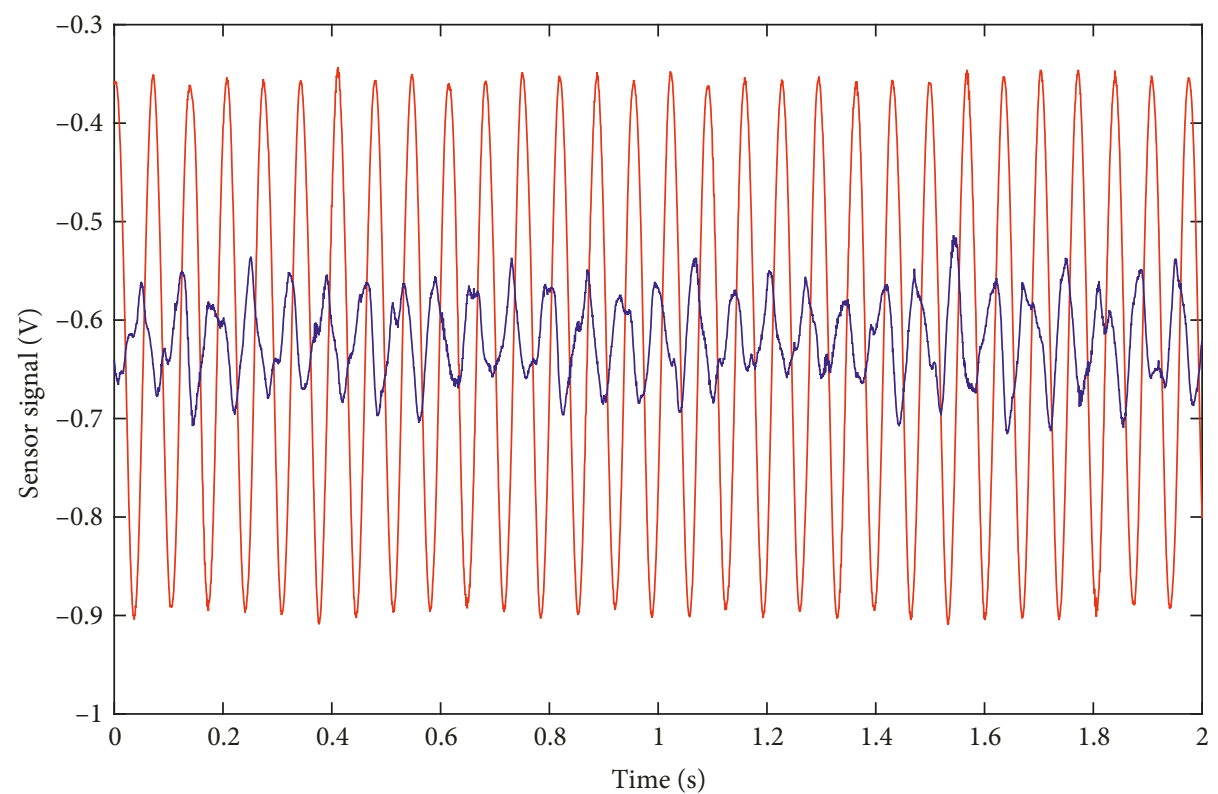

- Control OFF

Control ON

FIgURE 10: Comparison of time-domain signal with LQR control on/off.

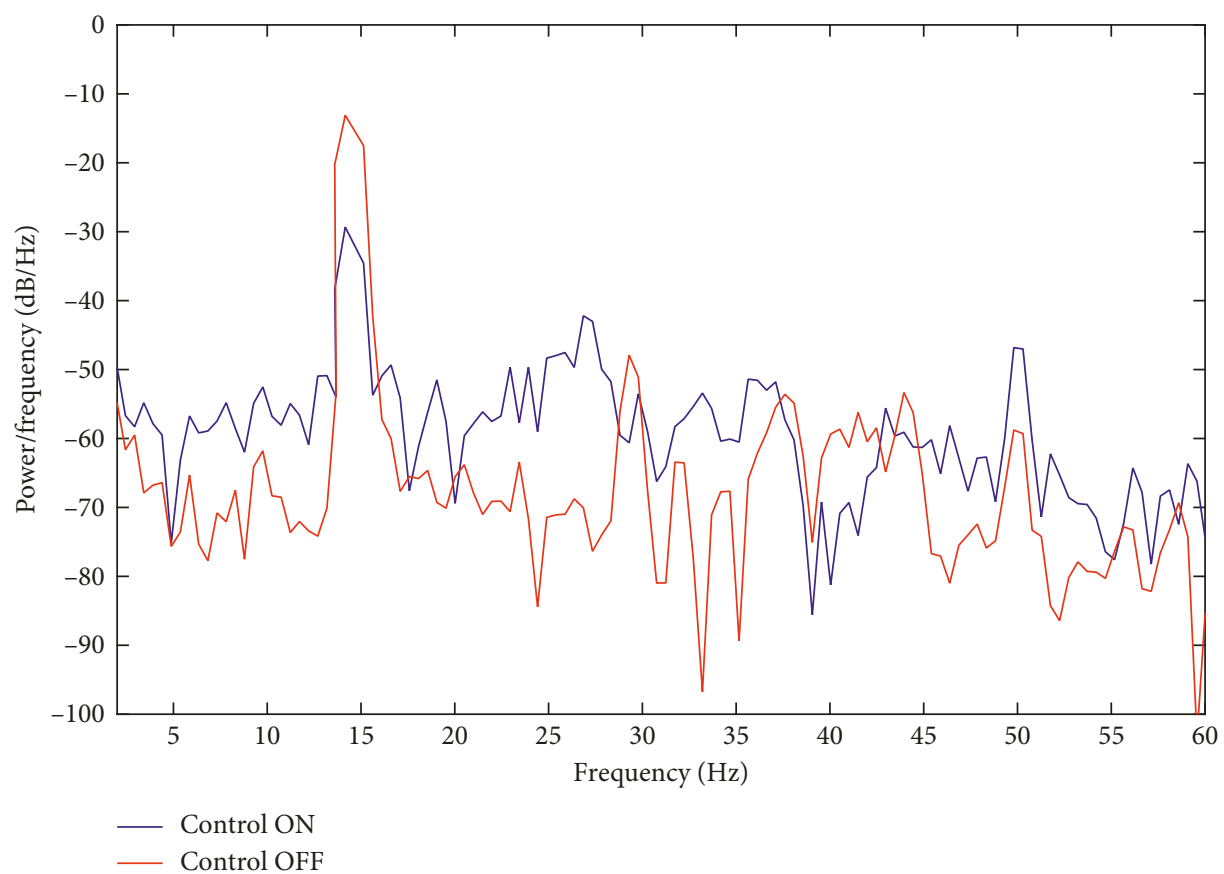

FIGURE 11: Comparison diagram of power spectrum with LQR control on/off.

TABLE 5: Comparison of three controllers.

\begin{tabular}{lccc}
\hline Types & $\begin{array}{c}\text { Maximum } \\
\text { damping percentage } \\
\text { in experiments }\end{array}$ & $\begin{array}{c}\text { Minimum } \\
\text { response } \\
\text { time }\end{array}$ & $\begin{array}{c}\text { Most notable } \\
\text { features }\end{array}$ \\
\hline PD & $81 \%$ & $1.5 \mathrm{~s}$ & Common and simple \\
NNPID & $88 \%$ & $1.2 \mathrm{~s}$ & Fast and self-tuning \\
LQR & $86 \%$ & $1.7 \mathrm{~s}$ & Saving energy \\
\hline
\end{tabular}

by China Aerodynamics Research and Development Center, which is confidential so cannot be made freely available. We are applying for a patent, which includes the details of the software program used to support the findings of this study so just a few parts of the program is available, which are on https://pan.baidu.com/s/1pHm-leBIDiN5zXXg0ua9bg (the code is ajqp). For more information, please contact Yuke Dai at ykdai@nuaa.edu.cn. 


\section{Conflicts of Interest}

The authors declare that there are no conflicts of interest regarding the publication of this paper.

\section{Acknowledgments}

This study was funded by the National Natural Science Foundation of China (No. 11872207) and Jiangsu Innovation Program for Graduate Education (No. KYLX15_0245).

\section{References}

[1] R. Wimmel, "Active electronic equipment DOF suspension for high loads as vibration, shock and quasi static forces," in Proceeding of the European Conference on Spacecraft Structures, Materials and Mechanical Testing, vol. 581, p. 32, Karen Fletcher, Noordwijk, The Netherlands, 2005.

[2] S. Balakrishna, D. Butler, M. Acheson, and E. White, "Design and performance of an active sting damper for the NASA common research model," in Proceedings of 49th AIAA Aerospace Sciences Meeting including the New Horizons Forum and Aerospace Exposition, pp. 953-963, American Institute of Aeronautics and Astronautics AIAA Associate, Orlando, FL, USA, January 2011.

[3] S. Balakrishna, H. Houlden, D. Butler et al., "Development of a wind tunnel active vibration reduction system," in Proceedings of 45th AIAA Aerospace Sciences Meeting and Exhibit, pp. 961-974, American Institute of Aeronautics and AstronauticsAIAA Associate, Reno, NV, USA, January 2007.

[4] G. Hefer, "ETW - a facility for high reynolds number testing," in Proceedings of IUTAM Symposium Transsonicum IV, Springer, Netherlands, 2003.

[5] H. Fehren, U. Gnauert, R. Wimmel, G. Hefer, and D. Schimanski, "Validation testing with the active damping system in the European transonic wind tunnel," in Proceedings of 39th AIAA Aerospace Sciences Meeting, p. 610, AIAA, Reno, NV, USA, January 2001.

[6] A. Nandi, S. Neogy, S. Bhaduri, and H. Irretier, "Vibration attenuation by a combination of a piezoelectric stack and a permanent magnet," Shock and Vibration, vol. 19, no. 4, pp. 719-734, 2012.

[7] Q. Zhang and Y. Jia, "Active noise feedback control using a neural network," Shock and Vibration, vol. 8, no. 1, pp. 15-19, 2001.

[8] Y. Meyer and M. Collet, "Active vibration isolation of electronic components by piezocomposite clamped-clamped beam," Mechanical Systems and Signal Processing, vol. 25, no. 5, pp. 1687-1701, 2011.

[9] J. Chen, X. Shen, F. Tu, and E. M. Qureshi, "Experimental research on an active sting damper in a low speed acoustic wind tunnel," Shock and Vibration, vol. 2014, Article ID 524351, 10 pages, 2014.

[10] K. L. Roger, G. E. Hodges, and L. Felt, "Active flutter suppression-a flight test demonstration," Journal of Aircraft, vol. 12, no. 6, pp. 551-556, 1975.

[11] T. Nestorović, N. Durrani, and M. Trajkov, "Experimental model identification and vibration control of a smart cantilever beam using piezoelectric actuators and sensors," Journal of Electroceramics, vol. 29, no. 1, pp. 42-55, 2012.

[12] T. Nestorović Trajkov, H. Köppe, and U. Gabbert, "Direct model reference adaptive control (MRAC) design and simulation for the vibration suppression of piezoelectric smart structures," Communications in Nonlinear Science and Numerical Simulation, vol. 13, no. 9, pp. 1896-1909, 2008.

[13] T. estorović, D. arinković, G. Chandrashekar, Z. Marinković, and M. Trajkov, "Implementation of a user defined piezoelectric shell element for analysis of active structures," Finite Elements in Analysis Design, vol. 52, pp. 11-22, 2012.

[14] Y. A. Zhuk, I. A. Guza, and C. M. Sands, "Monoharmonic approximation in the vibration analysis of a sandwich beam containing piezoelectric layers under mechanical or electrical loading," Journal of Sound and Vibration, vol. 330, no. 17, pp. 4211-4232, 2011.

[15] C. P. Young, T. G. Popernack, and B. B. Gloss, "National transonic facility model and model support vibration problems," in Proceedings of AIAA 16th Aerodynamic Ground Testing Conference, Seattle, WC, USA, June 1990.

[16] J. Fei, "Active vibration control of flexible steel cantilever beam using piezoelectric actuators," in Proceedings of ThirtySeventh Southeastern Symposium on System Theory, vol. 5, pp. 35-39, Los Alamitos, CA, USA, March 2005.

[17] L. Li, G. Song, and J. Ou, "Adaptive fuzzy sliding mode based active vibration control of a smart beam with mass uncertainty," Structural Control and Health Monitoring, vol. 18, no. 1, pp. 698-709, 2011.

[18] J. Lin and Y. B. Zheng, "Vibration suppression control of smart piezoelectric rotating truss structure by parallel neurofuzzy control with genetic algorithm tuning," Journal of Sound and Vibration, vol. 331, no. 16, pp. 3677-3694, 2012.

[19] J. Zhang, L. He, E. Wang, and R. Gao, "A LQR controller design for active vibration control of flexible structures," in Proceedings of 2008 IEEE Pacific-Asia Workshop on Computational Intelligence and Industrial Application Computational Intelligence and Industrial Application, PACIIA'08: PacificAsia Workshop, pp. 127-132, Wuhan, China, December 2008.

[20] M. S. Rechdaoui and L. Azrar, "Active control of secondary resonances piezoelectric sandwich beams," Applied Mathematics and Computation, vol. 216, no. 11, pp. 3283-3302, 2010.

[21] J.-E. Oh, S.-H. Park, J.-S. Hong, and J. Shin, “Active vibration control of flexible cantilever beam using piezo actuator and Filtered-X LMS algorithm," KSME International Journal, vol. 12, no. 4, pp. 665-671, 1998.

[22] J. Juang, M. Phan, L. G. Horta, and R. W. Longman, "Identification of observer/Kalman filter Markov parameters: theory and experiments," Journal of Guidance, Control, and Dynamics, vol. 16, no. 2, pp. 320-329, 1993.

[23] J. Juang and R. Pappa, "An Eigensystem realization algorithm for modal parameter identification and model reduction," Journal of Guidance, Control and Dynamics, vol. 8, no. 5, pp. 620-627, 1985. 


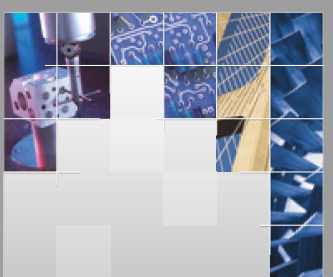

\section{Enfincering}
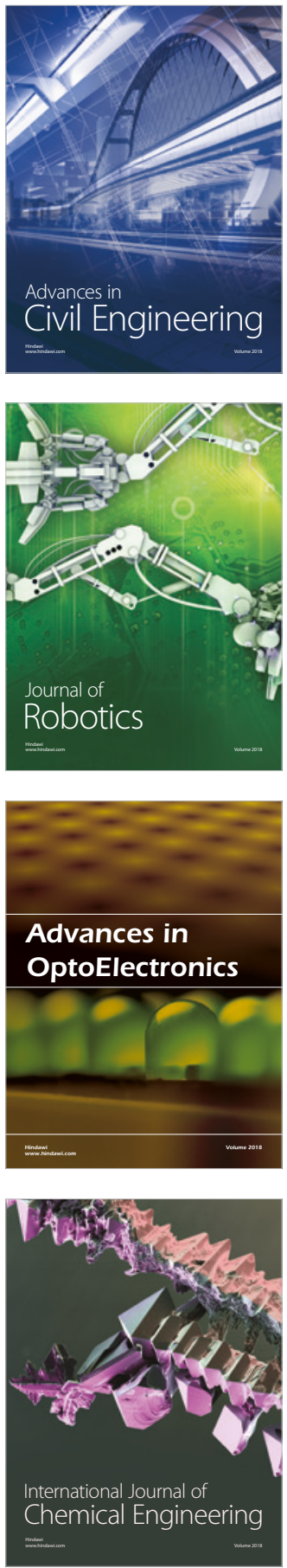

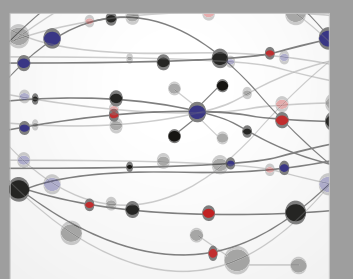

\section{Rotating \\ Machinery}

The Scientific World Journal

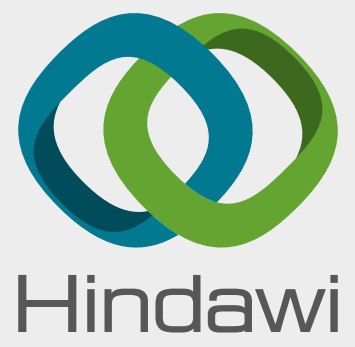

Submit your manuscripts at

www.hindawi.com
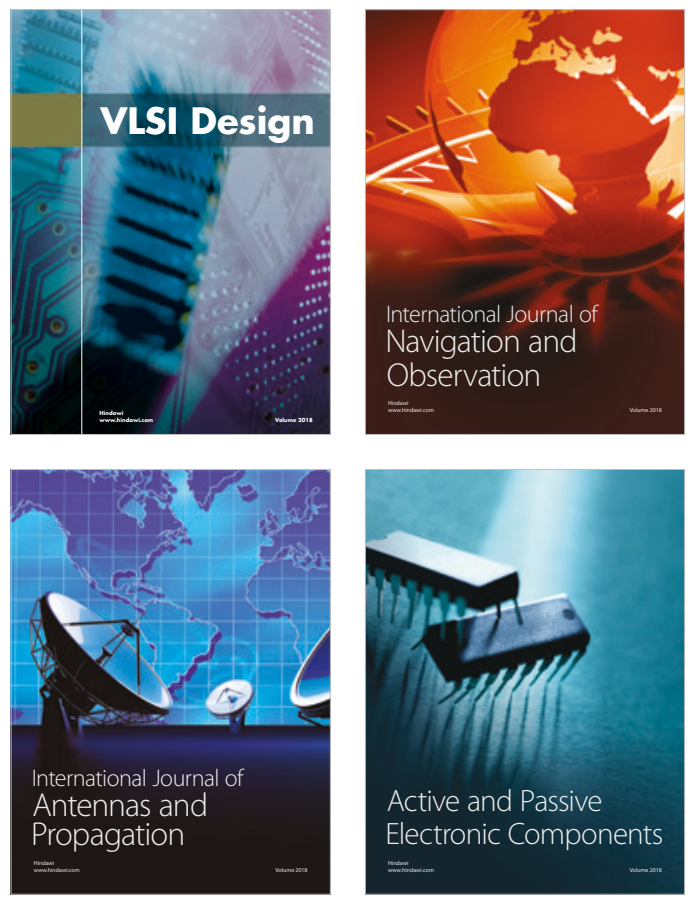
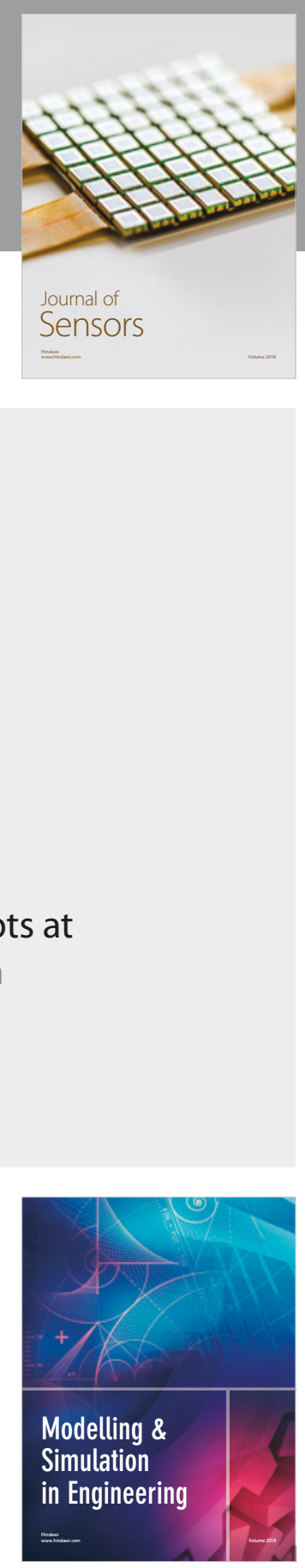

\section{Advances \\ Multimedia}
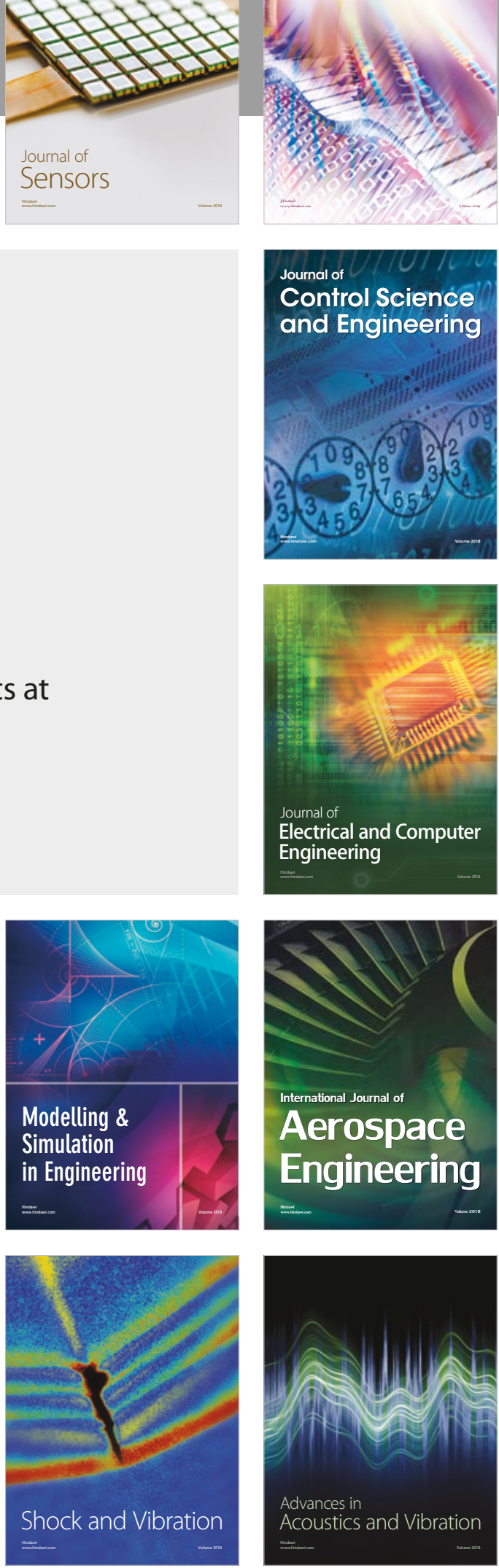